\title{
Intraoperative visualization of plasmon resonant liposomes using augmented microscopy
}

Jeffrey R. Watson Summer Garland Marek Romanowski 


\title{
Intraoperative Visualization of Plasmon Resonant Liposomes Using Augmented Microscopy \\ Jeffrey R Watson, Summer Garland, Marek Romanowski ${ }^{*}$ \\ Department of Biomedical Engineering, University of Arizona, Tucson, AZ \\ *marekrom@email.arizona.edu; phone 1520 626-1578; fax 1520 626-8726
}

\begin{abstract}
Plasmon resonance associated with nanoparticles of gold can enable photothermal ablation of tissues or controlled drug release with exquisite temporal and spatial control. These technologies may support many applications of precision medicine. However, clinical implementations of these technologies will require new methods of intraoperative imaging and guidance. Near-infrared laser surgery is a prime example that relies on improved image guidance. Here we set forth applications of augmented microscopy in guiding surgical procedures employing plasmon resonant gold-coated liposomes. Absorption of near-infrared laser light is the first step in activation of various diagnostic and therapeutic functions of these novel functional nanoparticles. Therefore, we demonstrate examples of near-infrared visualization of the laser beam and gold-coated liposomes. The augmented microscope proves to be a promisingimage guidance platform for a range of image-guided medical procedures.
\end{abstract}

Keywords: Augmented microscopy, near-infrared laser, plasmon resonant, fluorescence, gold nanoparticles, liposomes, intraoperative imaging, image guided surgery

\section{INTRODUCTION}

State of the art image guidance technologies and therapeutic strategies are of paramount importance for improving the outcomes of surgical procedures. Brain cancer has a high morbidity in part because of the difficult and incomplete resection margins. ${ }^{1}$ This can be attributed to two main impediments: inability to visualize the tumor tissue completely, and inadequate techniques for resection of the tumor tissue. Glioblastoma (GBM), considered the most aggressive and difficult to treat brain cancer, is highly invasive and grows diffusely with 'finger-like' projections. While there is a positive correlation between better resection margins and improved prognosis, identifying tumor margins is extremely diffciult. ${ }^{2}$ The diffusivity of GBM creates the need for better diagnostic imaging techniques that can highlight these margins. Additionally, there also exists a need for improved treatment strategies to aid in more complete removal or even eradication of the tumor tissue. Laser surgery in combination with nanotechnology can potentially improve treatment for brain cancer. Nanotechnology has yield new materials of unique properties. For example, gold nanoparticles have been shown to absorb energy of near-infrared (NIR) light and enable thermal ablation for biomedical applications. Consequently, there has been increased focus on using near-infrared light for surgical image guidance and therapy to enhance visualization of abnormal or pathological structures and ablate tissue respectively. ${ }^{2-10}$ NIR light is advantageous for the transparency of biological tissues in this spectral range, and introducing NIR light does not affect the perceived colors in the visible spectrum. Currently, surgical imaging technologies have limited capabilities to work with images simultaneously in the NIR and visible spectrum. Leading surgical microscopes, like those from Leica or Zeiss, provide NIR capabilities as an 'add-on' functionality, but these microscopes are not optimized for real-time NIR imaging or NIR light sensitivity balanced with visible light sensitivity. Therefore, there is a need to develop more effective imaging technologies that can better visualize, and be more sensitive to, the NIR light. We are developing a surgical augmented microscope to provide concurrent visualization of the surgical field with images derived from a NIRsensitive camera for guiding surgical procedures in the brain that use NIR laser light and novel gold-coated nanoparticles.

Standard gold nanoparticles are typically prepared in the size range of 50-100 $\mathrm{nm}$ and there are still concerns regarding clearance and toxicity. ${ }^{11}$ To address clearance and toxicity relevant issues, we investigate gold-coated, plasmon resonant liposomes.$^{12}$ These nanoparticles are synthesized from lipids and have small clusters of gold, less than $5 \mathrm{~nm}$ in size, reduced onto the surface. We expect that these nanoparticles will degrade via normal biological processes to clusters small enough for renal excretion. In addition to addressing clearance issues, gold-coated liposomes are uniquely capable

Molecular-Guided Surgery: Molecules, Devices, and Applications III, edited by Brian W. Pogue, Sylvain Gioux, Proc. of SPIE Vol. 10049, 100490L · (C) 2017 SPIE · CCC code: 1605-7422/17/\$18 - doi: 10.1117/12.2248706 
of activated drug delivery via modulated NIR laser. ${ }^{13,14}$ Because of their various modes of interaction with NIR light, gold-coated liposomes present a unique choice for visualization and guidance under the surgical augmented microscope.

This paper reviews our continuing work toward a surgical augmented microscope for guiding NIR laser surgery with gold-coated, plasmon resonant liposomes. We discuss a new optical module that will enable augmentation within a surgical stereomicroscope for better visualizing contrast agents and NIR laser beams concurrently with presentation of the surgical field.

\section{MATERIALS AND METHODS}

All animal procedures performed in this study were approved by the Institutional Animal Care and Use Committee at the University of Arizona.

\subsection{Surgical Augmented Microscope}

Current implementation of the augmented microscope is based on our work presented earlier. ${ }^{9,10}$ It uses a surgical ENT microscope body and stand (Prima ENT, Labo America, Inc., Fremont, CA) (Fig. 1a) and meets optical and ergonomic standards that are expected in the clinical setting (i.e., working distance, depth of focus, white light flux, maneuverability). This microscope retains the Galilean optical design, with two parallel optical paths for stereovision, which enables a modular augmentation module to be inserted into the optical path. Here, we are developing a complete, single module that will enable stereoscopic augmentation in the new surgical microscope (Fig 1b). The new module contains the excitation source optics for NIR fluorescence applications. It uses NIR optics and NIR sensitive camera (ImageM EM-CCD, Hamamatsu, Hamamatsu City). The module employs a miniature digital micromirror device (DMD) picoprojector (DLP LightCrafter Display 2010 EVM, Texas Instruments, Dallas, TX) to inject the synthetic image into the optical path of the microscope. A patent pending technology for combining real and synthetic images uses a spatial light modulator, presented in Poster 10049-42.

\subsection{Cerebrovascular Angiography with Plasmon Resonant Gold-Coated Liposomes}

\subsubsection{Gold-Coated Liposomes with NIR Fluorescence}

NIR-fluorescent gold-coated liposomes were prepared using standard techniques and loaded with a hydrophobic NIR fluorescent dye (IR792, Sigma-Aldrich, St. Louis, MO). ${ }^{13}$ Briefly, a mixture of lipids with a NIR dye was dried overnight using a vacuum cold trap and then rehydrated with phosphate buffered saline. Freeze/thaw cycles were subsequently performed followed by high pressure extrusion through $100 \mathrm{~nm}$ pore size filters. Gold coating was performed by reducing gold-chloride onto the surface of the liposomes in the presence of ascorbic acid. The presence of plasmon resonance was confirmed by absorption spectra (Cary5, Santa Clara, CA), and the size of nanoparticles was confirmed via dynamic light scattering (ZetaSizer Nano ZS90, Malvern, UK). The gold-coated liposomes were prepared at $30 \mathrm{mM}$ lipid concentration with 0.1 mole percent of the NIR dye.

\subsubsection{Cerebrovascular Angiography}

Angiography was performed in a rat model of an open craniotomy. The animal was anesthetized (1-3\% isoflurane, Henry Schein, Melville, NY) and monitored visually and using tow pinch technique periodically during surgery. Immediately the tail vein was cannulated for vascular access. Under the augmented microscope, approximately $2 \times 5 \mathrm{~mm}$ craniotomy was performed on the right lateral side of the superior central sulcus between bregma and lambda using a high-speed drill. The dura was carefully removed using microsurgical technique to fully expose brain vasculature and any bleeding was controlled. The gold-coated liposomes with NIR dye were injected $(0.5 \mathrm{~mL})$ through the tail vein cannula while the surgical field and NIR fluorescence were observed within the oculars of the augmented microscope and recorded for further analysis.

\subsection{NIR Laser Guidance Using Augmented Microscope}

The augmented microscope was used to observe an ultrafast Ti:Sapphire NIR laser beam to develop an experimental protocol for NIR laser ablation with gold-coated liposomes. We launched a Ti:Sapphire laser beam (MaiTai, Spectra Physics, Santa Clara, CA) at $<5 \mathrm{~mW}$ average power into a hollow core photonic crystal fiber (PCF) (HC-800B, Thorlabs, Newton, NJ) using a precision single mode fiber launch stage (MBT612D, Thorlabs). A FC type connector (30140E1, Thorlabs) and appropriate beam collimator (F230FC-780, Thorlabs) was attached to the distal end of the fiber. The NIR laser beam was observed using the augmented microscope and images recorded for analysis. 


\section{RESULTS}

\subsection{Surgical Augmented Microscope}

A custom augmentation module was designed using SolidWorks and machined at the University of Arizona Aerospace and Mechanical Engineering machine shop (Fig 1b). This module houses the necessary optics and technology to enable augmentation within the new surgical microscope. A custom 3D printed plastic mold holds and aligns the optical assembly and components for enabling augmentation. The new augmentation module is designed to allow for maneuverability of the microscope head while minimizing added weight to the pantographic arm.
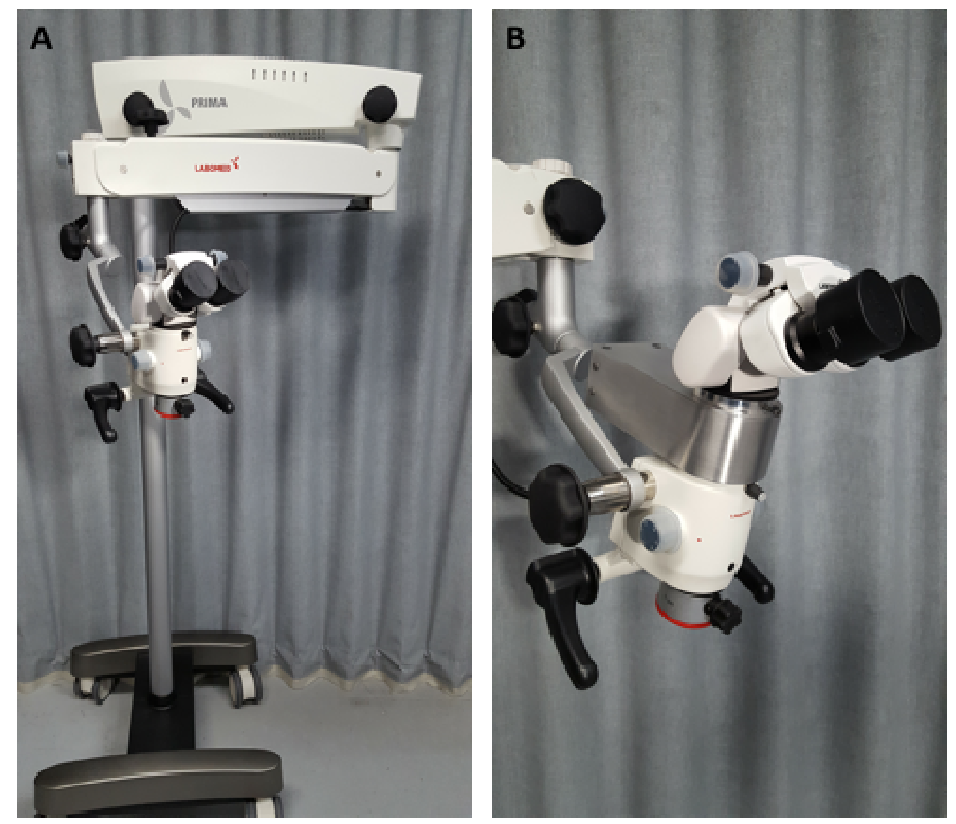

Figure 1: Surgical augmented microscope fitted with preliminary augmentation module between microscope body and ocular head. A. Labomed surgical ENT microscope being used for the updated augmented microscope; B. surgical ENT microscope with new augmentation module inserted between microscope body and ocular head.

\subsection{Cerebrovascular Angiography with Plasmon Resonant Gold-Coated Liposomes}

The NIR-fluorescent gold-coated liposomes were used for angiography and visualized within an open craniotomy on a rat under the augmented microscope (Fig 2). The NIR-fluorescent gold-coated liposomes are larger than a standard molecular dye making it less likely to extravasate thereby reducing background signal over subsequent injections. Fluorescent signal was confined to the cerebral vasculature. A strong signal was observed from the fully exposed brain vasculature. A noticeable signal was also observed through the cranium directly above the superior sagittal sinus (Fig 2b). There was minimal scattered fluorescence signal within the craniotomy maintaining clear and crisp fluorescence signal. 

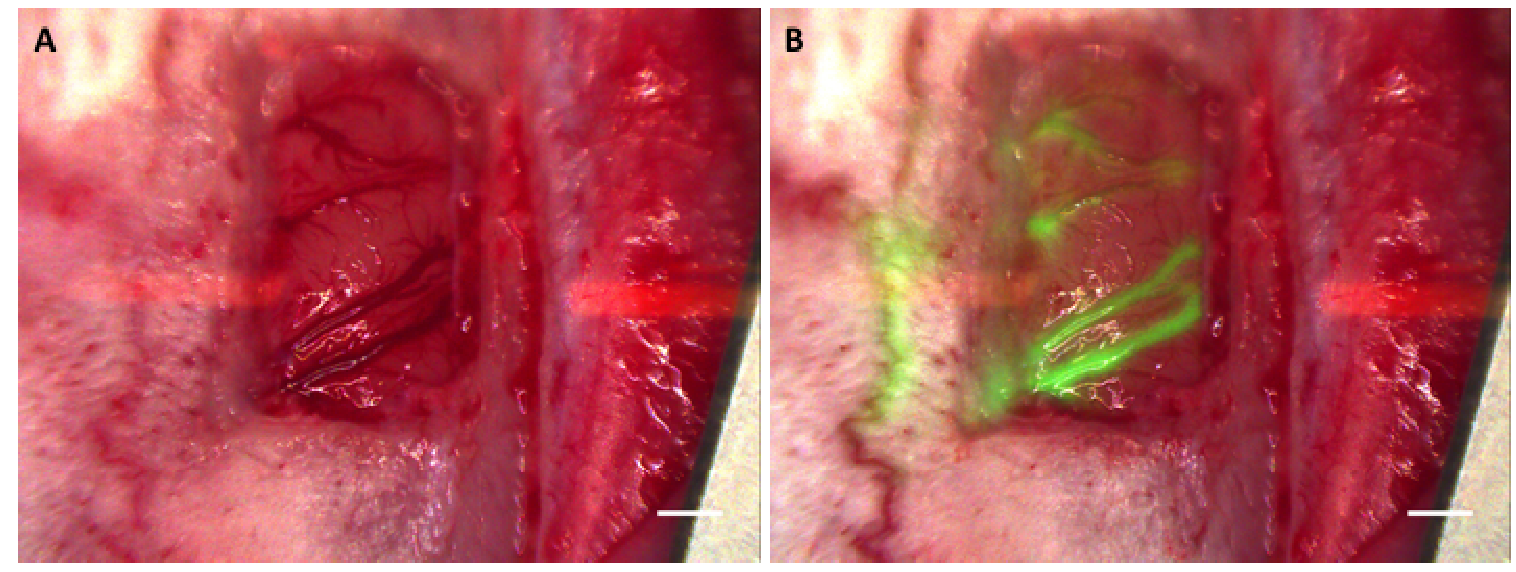

Figure 2: Augmented vascular angiography with gold-coated liposome nanoparticles synthesized with IR792 fluorescent dye as NIR contrast agent. A. Image shows without augmentation enabled, B. Image shows with augmentation enabled. Horizontal line seen in the right image is a result of visible camera artifact. Scale bar $1 \mathrm{~mm}$.

\subsection{NIR Laser Guidance Using Augmented Microscopy}

We visualized a Ti:Sapphire laser beam under the augmented microscope to demonstrate the ability to guide NIR laser surgery procedures (Fig 3). The microscope enables complete visualization of the laser beam and surrounding spatial queues as seen in Fig 3. The fiber collimator produces a $5 \mathrm{~mm}$ diameter beam that is clearly visible using the augmented microscope. Location of the NIR laser beam or surrounding spatial features would not be possible without the augmentation enabled.
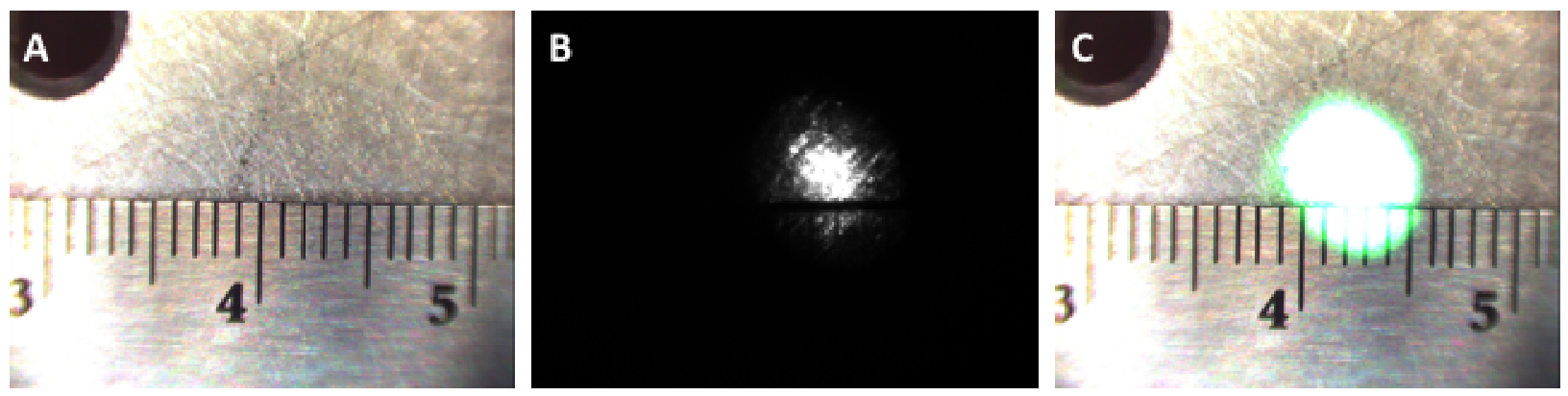

Figure 3: NIR laser guidance using augmented microscope. Visualization of $820 \mathrm{~nm}$ Ti:Sapphire laser from a hollow core PCF. A. no augmentation doesn't allow for visualization of the laser beam; B. NIR view only shows the laser beam without visible spatial features; C. augmented view shows both NIR laser beam (false colored green) and visible spatial features.

\section{DISCUSSION}

The augmented microscope has been extensively evaluated and characterized previously. From prior experiments, we identified certain limitations and concerns that needed to be addressed before moving to clinical research models. The first prototype used a standard, modular Olympus SZX7 stereomicroscope that had a marginal working distance, on the order of $10 \mathrm{~cm}$. Current surgical microscopes used in the neurosurgical operating room have working distances greater than $30 \mathrm{~cm}$. This is essential for ergonomics and functionality during the surgical procedures and enables enough room for working with tools underneath the objective. The longer working distance requires a lower numerical aperture objective that also produces a large depth of field. The large depth of field is also imperative for surgical applications to ensure that all structures within the field of view are in full focus. However, the lower numerical aperture requires a very intense illumination of the field of view to compensate for lower light throughput. The initial prototype augmented microscope, under full surgical illumination and maximum microscope light output, had an OLED projector that was unable to produce images with adequate contrast against white light within the microscope. These primary concerns are being addressed via new module design and hardware in the updated surgical augmented microscope. 
The current prototype combines all the necessary parts into one complete module for insertion between ocular head and microscope body of a surgical microscope. To address the inadequate contrast between augmentation images and white light observed in the first prototype, we have fitted the new module with a DMD picoprojector, capable of high luminance and excellent contrast that can more closely match the intensity of surgical illumination. This produces better quality augmentation within the oculars while retaining full surgical illumination. The surgical augmented microscope uses an EMCCD sensor for detecting NIR light to retain sensitivity required in proposed imaging applications.

The gold-coated liposomes were clearly visualized within brain vasculature demonstrating their possible use as a vascular contrast agent. In contrast with molecular dyes (e.g. indocyanine green), we observed little extravascular background signal. The relatively larger size of the gold-coated liposomes impedes extravasation, even within diseased vasculature in the brain. The fluorescence signal seen above the superior sagittal sinus indicates the deep detection depth through the cranium.

These observations point to several potential clinical applications of the augmented microscopy. The augmented microscope visualized near-infrared laser light while still maintaining the ability to monitor surrounding spatial cues. When working with gold-coated, plasmon resonant liposomes the NIR laser light can be introduced to activate therapies for subsequent therapeutic processes. Gold-coated liposomes can either release their contents, heat up and ablate tissue, or both. NIR fluorescent dyes can be added to liposomes to track their distribution and evaluate the therapeutic process. When accumulated at a tumor site, the nanoparticles could be illuminated with NIR laser light during surgery to improve spatial control and accuracy of the therapeutic process. In another area of potential clinical applications, the delivery and monitoring of ultrafast laser pulses under the augmented microscope may enable implementation of non-thermal laser ablation technologies for surgery.

The surgical augmented microscope provides a promising image guidance technology for visualizing NIR procedures. The simple and ergonomic integration of the module into a surgical microscope will be advantageous to the surgical community and provide easy access to augmented imaging. 


\section{REFERENCES}

[1] American Brain Tumor Association, http://www.abta.org/about-us/news/brain-tumor-statistics (January 2017).

[2] Y. Li et al., "The influence of maximum safe resection of glioblastoma on survival in 1229 patients: Can we do better than gross-total resection?," J. Neurosurg. (2016).

[3] R. M. Schols, N. J. Connell, and L. P. S. Stassen, "Near-Infrared Fluorescence Imaging for Real-Time Intraoperative Anatomical Guidance in Minimally Invasive Surgery: A Systematic Review of the Literature," World J. Surg. 39(5), 1069-1079, Springer International Publishing (2015) [doi:10.1007/s00268-014-2911-6].

[4] A. L. Vahrmeijer et al., "Image-guided cancer surgery using near-infrared fluorescence," Nat. Rev. Clin. Oncol. 10(9), 507-518, Nature Research (2013) [doi:10.1038/nrclinonc.2013.123].

[5] S. Gioux, H. S. Choi, and J. V. Frangioni, "Image-Guided Surgery Using Invisible Near-Infrared Light: Fundamentals of Clinical Translation," http://dx.doi.org/10.2310/7290.2010.00034, SAGE PublicationsSage CA: Los Angeles, CA (2010) [doi:10.2310/7290.2010.00034].

[6] A. V. DSouza et al., "Review of fluorescence guided surgery systems: identification of key performance capabilities beyond indocyanine green imaging," J. Biomed. Opt. 21(8), 80901, International Society for Optics and Photonics (2016) [doi:10.1117/1.JBO.21.8.080901].

[7] J. T. Elliott et al., "Review of fluorescence guided surgery visualization and overlay techniques," Biomed. Opt. Express 6(10), 3765, Optical Society of America (2015) [doi:10.1364/BOE.6.003765].

[8] André M. Gobin et al., "Near-Infrared Resonant Nanoshells for Combined Optical Imaging and Photothermal Cancer Therapy," American Chemical Society (2007) [doi:10.1021/NL070610Y].

[9] J. R. Watson et al., "Augmented microscopy: real-time overlay of bright-field and near-infrared fluorescence images," J. Biomed. Opt. 20(10), 106002, International Society for Optics and Photonics (2015) [doi:10.1117/1.JBO.20.10.106002].

[10] N. L. Martirosyan et al., "Integration of Indocyanine Green Videoangiography With Operative Microscope," Neurosurgery 11, 252-258 (2015) [doi:10.1227/NEU.0000000000000681].

[11] M. F. Hornos Carneiro and F. Barbosa, "Gold nanoparticles: A critical review of therapeutic applications and toxicological aspects," J. Toxicol. Environ. Heal. Part B 19(3-4), 129-148, Taylor \& Francis (2016) [doi:10.1080/10937404.2016.1168762].

[12] T. S. Troutman, J. K. Barton, and M. Romanowski, "Biodegradable Plasmon Resonant Nanoshells," Adv. Mater. 20(13), 2604-2608, WILEY VCH Verlag (2008) [doi:10.1002/adma.200703026].

[13] S. J. Leung, M. C. Bobnick, and M. Romanowski, "Plasmon resonant gold-coated liposomes for spectrally controlled content release.," Proc. SPIE--the Int. Soc. Opt. Eng. 7577, NIH Public Access (2010).

[14]T. S. Troutman, S. J. Leung, and M. Romanowski, "Light-Induced Content Release from Plasmon-Resonant Liposomes,” Adv. Mater. 21(22), 2334-2338, WILEY VCH Verlag (2009) [doi:10.1002/adma.200900018]. 